\title{
REGULARITY OF CERTAIN SMALL SUBHARMONIC FUNCTIONS
}

BY

P. C. FENTON

ABSTraCt. Suppose that $u$ is subharmonic in the plane and that $\lim _{r \rightarrow \infty} B(r) /(\log r)^{2}=\sigma<\infty$. It is known that, given $\varepsilon>0$, there are arbitrarily $\overline{l a r g e}$ values of $r$ such that $A(r)>B(r)-(\sigma+\varepsilon) \pi^{2}$. The following result is proved. Let $u$ be subharmonic and let $\sigma$ be any positive number. Then either $A(r)>B(r)-$ $\pi^{2} \sigma$ for certain arbitrarily large values of $r$ or, if this is false, then

$$
\lim _{r \rightarrow \infty}\left(B(r)-\sigma(\log r)^{2}\right) / \log r
$$

exists and is either $+\infty$ or finite.

1. Introduction. Let $u(z)$ be subharmonic in the plane and define

$$
\begin{aligned}
& B(r)=B(r, u)=\max _{|z|=r} u(z), \\
& A(r)=A(r, u)=\inf _{|z|=r} u(z) .
\end{aligned}
$$

In [3] the following result is proved.

TheOREM A. Let $p>1$ be given and suppose that $u(z)$ is subharmonic in the plane and satisfies

$$
\lim _{r \rightarrow \infty} \frac{B(r)}{(\log r)^{p}}=\sigma<\infty
$$

Then, given $\varepsilon>0$,

$$
A(r)>B(r)-(\sigma+\varepsilon) \operatorname{Re}\left\{(\log r)^{p}-(\log r+i \pi)^{p}\right\}
$$

for $r$ outside an exceptional set $E$ for which

$$
\lim _{r \rightarrow \infty} \frac{(p-1)}{(\log r)^{p-1}} \int_{E \cap[1, r]} \frac{(\log r)^{p-2}}{t} d t \leqslant \frac{\sigma}{\sigma+\varepsilon} .
$$

Theorem $A$ is related to certain results of P. D. Barry. (See Theorem 4 and the remarks in $\$ 7.4$ of [1].) With Kjellberg's version of the $\cos \pi \lambda$ Theorem [5], [6] in view we might expect that functions extremal for Theorem A would have some kind of regular asymptotic behaviour. In this direction we shall prove

Theorem 1. Suppose that $u(z)$ is subharmonic in the plane and that $\sigma$ is any positive number. Then either

$$
A(r)>B(r)-\pi^{2} \sigma
$$

\footnotetext{
Received by the editors April 24, 1979 and, in revised form, December 10, 1979. AMS (MOS) subject classifications (1970). Primary 31A05. 
for certain arbitrarily large values of $r$ or, if this is not the case, then

$$
\alpha=\lim _{r \rightarrow \infty} \frac{B(r)-\sigma(\log r)^{2}}{\log r}
$$

exists and is either $+\infty$ or finite.

This result corresponds to $p=2$ in Theoram A. It seems likely that, for the case of general $p$, the limit of (1.3) could be replaced by

$$
\lim _{r \rightarrow \infty} \frac{B(r)-\sigma(\log r)^{p}}{(\log r)^{p-1}}>-\infty .
$$

EXAMPLE. Any value of $\alpha$ admitted by Theorem 1 can in fact occur. Write $z=r e^{i \theta}$, where $-\pi<\theta \leqslant \pi$. In the case $\alpha=+\infty$ we may let $u(z)=r \cos \theta$. If $\alpha=0$ we set

$$
\begin{aligned}
& u_{0}(z)=0, \quad r \leqslant 1, \\
& u_{0}(z)=\max \left(\sigma(\log r)^{2}-\sigma \theta^{2}, 0\right), \quad r>1 .
\end{aligned}
$$

Evidently $u_{0}(z)$ is continuous and subharmonic except possibly on the segment $r>1$ of the negative real axis, since $\sigma(\log r)^{2}-\sigma \theta^{2}=\operatorname{Re} \sigma(\log r+i \theta)^{2}$ is harmonic except for negative real $z$. If we write $\theta_{1}, \theta_{2}$ for the values of $\arg z$ which satisfy respectively

$$
-2 \pi<\theta_{1}<0, \quad 0<\theta_{2}<2 \pi
$$

and set

$$
u_{1}(z)=\sigma(\log r)^{2}-\sigma \theta_{1}^{2}, \quad u_{2}(z)=\sigma(\log r)^{2}-\sigma \theta_{2}^{2},
$$

then $u_{1}$ and $u_{2}$ are harmonic near the negative real axis and so

$$
u_{0}(z)=\max \left(u_{1}(z), u_{2}(z)\right)
$$

is subharmonic there and so everywhere in the plane. Also, for any real $\alpha$, we define $c$ by $2 \sigma \log c=\alpha$, and set $u(z)=u_{0}(c z)-\sigma(\log c)^{2}$. Then

$$
\begin{aligned}
& B(r, u)=\sigma(\log r)^{2}+\alpha \log r, \quad r>1 / c, \\
& A(r, u)=B(r, u)-\pi^{2} \sigma, \quad r>e^{\pi} / c,
\end{aligned}
$$

so that (1.2) fails and

$$
\frac{B(r, u)-\sigma(\log r)^{2}}{\log r}=\alpha, \quad r>1 / c .
$$

From the Riesz representation theorem for subharmonic functions it follows that there is a unique nonnegative measure $\mu$ defined on all bounded, Borel measurable subsets of the plane such that, if $R$ is a given positive number,

$$
u(z)=h_{R}(z)+\int_{|\zeta|<R} \log \left|1-\frac{z}{\zeta}\right| d \mu(\zeta)
$$

for $|z|<R$, where $h_{R}(z)$ is harmonic in $|z|<R$. In the proof it is assumed that $u$ is harmonic at 0 but this may be achieved without loss of generality to our results by replacing $u$ in a disc about 0 by the Poisson integral of its boundary values on the 
disc. Throughout the paper we shall assume that $u(0)=0$, as we may do without affecting the generality of our results. We define $\mu^{*}(r)=\mu(\{z:|z|<r\})$ for $r>0$.

TheOREM 2. Suppose that $u(z)$ is subharmonic in the plane and that $\sigma$ is a positive number such that

$$
A(r) \leqslant B(r)-\pi^{2} \sigma
$$

for all large $r$. Suppose further that the limit $\alpha$ of (1.3) is finite. Let

$$
u_{1}(z)=\int_{|\zeta|<\infty} \log \left|1+\frac{z}{|\zeta|}\right| d \mu(\zeta)=\int_{0}^{\infty} \log \left|1+\frac{z}{t}\right| d \mu^{*}(t)
$$

and define $B_{1}(r)=\max _{|z|=r} u_{1}(z)$. Then

$$
\lim _{r \rightarrow \infty} \frac{B_{1}(r)-\sigma(\log r)^{2}}{\log r}=\alpha .
$$

THEOREM 3. Under the conditions of Theorem 2

$$
\lim _{r \rightarrow \infty}\left(\mu^{*}(r)-2 \sigma \log r\right)=\alpha .
$$

The first part of the paper is devoted to showing that, under the conditions expressed in the second alternative of Theorem $1, \mu^{*}(r)=O(\log r)$ when

$$
\underline{\lim }\left(B(r)-\sigma(\log r)^{2}\right) / \log r<\infty .
$$

This is rather more drawn out than might be expected due to certain tiresome modifications to $u$ that seem to be necessary in the subsequent parts of the proof. In $\$ 6$ and $\$ 7$ the growth properties of $u$ and $u_{1}$ are considered and the theorems are proved more or less together.

2. Decomposition of $u$. In [2] Barry has put into subharmonic form results derived by Kjellberg [5, pp. 190-192] in the case $u(z)=\log |f(z)|$, where $f$ is an entire function. Some of these are as follows.

With $\mu^{*}(t)=\mu(|z|<t)$ define

$$
\begin{aligned}
& u_{1}(z, R)=\int_{|\zeta|<R} \log \left|1-\frac{z}{\zeta}\right| d \mu(\zeta), \\
& u_{2}(z, R)=\int_{|\zeta|<R} \log \left|1+\frac{z}{|\zeta|}\right| d \mu(\zeta)=\int_{0}^{R} \log \left|1+\frac{z}{t}\right| d \mu^{*}(t), \\
& u_{3}(z, R)=u(z)-u_{1}(z, R) .
\end{aligned}
$$

Then, with $B_{j}(r, R)=\max _{|z|=r} u_{j}(z, R), A_{j}(r, R)=\inf _{|z|=r} u_{j}(z, R), j=1,2,3$,

$$
A_{2}(r, R) \leqslant A_{1}(r, R) \leqslant B_{1}(r, R) \leqslant B_{2}(r, R) ;
$$

and

$$
-\frac{4 r}{R} B(2 R)<A_{3}(r, R)<B_{3}(r, R)<\frac{4 r}{R} B(2 R) .
$$

for $0 \leqslant r \leqslant \frac{1}{2} R$. 
We note finally the subharmonic analogue of Jensen's Theorem [4, p. 473]: for $r>0$

$$
u(0)+\int_{0}^{r} \log \frac{r}{t} d \mu^{*}(t)=\frac{1}{2 \pi} \int_{0}^{2 \pi} u\left(r e^{i \theta}\right) d \theta \leqslant B(r) .
$$

Concerning $u_{2}(z, R)$ we have the following lemma.

LeMma 1. Let $R_{1}, R_{2}$ and $R$ be positive numbers satisfying $R_{1}<R_{2}<R$. Then

$$
\begin{aligned}
\int_{R_{1}}^{R_{2}} \frac{A_{2}(t, R)-B_{2}(t, R)}{t} d t & \\
= & \mu^{*}(R) \int_{0}^{\pi} \operatorname{Arg}\left(1-\frac{R_{2}}{R} e^{i \theta}\right) d \theta-\int_{0}^{R} \frac{\mu^{*}(t)}{t} \log \left|\frac{t+R_{2}}{t-R_{2}}\right| d t \\
& -\mu^{*}(R) \int_{0}^{\pi} \operatorname{Arg}\left(1-\frac{R_{1}}{R} e^{i \theta}\right) d \theta+\int_{0}^{R} \frac{\mu^{*}(t)}{t} \log \left|\frac{t+R_{1}}{t-R_{1}}\right| d t .
\end{aligned}
$$

Also

$$
\begin{aligned}
I\left(R_{1}, R_{2}, R\right)= & \int_{R_{1}}^{R_{2}} \frac{d s}{s} \int_{0}^{s} \frac{A_{2}(t, R)-B_{2}(t, R)}{t} d t \\
= & \mu^{*}(R) \int_{R_{1}}^{R_{2}} \frac{d s}{s} \int_{0}^{\pi} \operatorname{Arg}\left(1-\frac{s}{R} e^{i \theta}\right) d \theta \\
& -\int_{0}^{R} \frac{\mu^{*}(t)}{t} d t \int_{R_{1}}^{R_{2}} \frac{1}{s} \log \left|\frac{s+t}{s-t}\right| d s
\end{aligned}
$$

The first part is contained in the proof of the Lemma in [3] and the second part follows immediately from the first on integration.

3. Preliminaries. To prove the theorems we assume that for all large $r$

$$
A(r) \leqslant B(r)-\pi^{2} \sigma
$$

and that

$$
\alpha=\lim _{r \rightarrow \infty} \frac{B(r)-\sigma(\log r)^{2}}{\log r}<+\infty
$$

and aim to prove the existence of the finite limit (1.3). The first step is to show that (3.1) and (3.2) together imply

$$
\mu^{*}(r)=O(\log r) \text { as } r \rightarrow \infty
$$

and in order to do this we assume that

$$
\varlimsup_{r \rightarrow \infty} \frac{\mu^{*}(r)}{\log r}=+\infty
$$

and deduce a contradiction. We have 
LEMMA 2. Suppose that (3.1), (3.2) and (3.4) hold. Then there exists a subharmonic function $U(z)$ which satisfies the following conditions:

$$
\text { (i) } \begin{aligned}
\frac{\lim }{r \rightarrow \infty} \frac{B(r, U)-\sigma(\log r)^{2}}{\log r} & <-A<-\frac{4 \sigma}{\pi^{2}}\left(\pi^{2}+90\right) ; \\
\text { (ii) } A(r, U) & =B(r, U)-\pi^{2} \sigma
\end{aligned}
$$

holds for $e^{\pi} \leqslant r \leqslant r_{0}$ and for $2 r_{0} \leqslant r<\infty$, for some $r_{0}>e^{\pi}$;

$$
\text { (iii) } \varlimsup_{r \rightarrow \infty} \frac{\mu^{*}(r, U)}{\log r}=\infty \text {. }
$$

Set $v_{1}(z)=\max \left\{u(z), B(|z|)-\pi^{2} \sigma\right\}$. Since $B(|z|)$ is subharmonic [7, §3.20] so also is $v_{1}(z)$. Also, since $v_{1}$ and $u$ have the same maximum on each circle about the origin, (3.2) holds for $v_{1}$; and it follows from (2.6) that (3.4) is equivalent to

$$
\varlimsup_{r \rightarrow \infty} \frac{1}{(\log r)^{2}} \int_{0}^{2 \pi} u\left(r e^{i \theta}\right) d \theta=\infty,
$$

so that (3.4) persists likewise for $v_{1}$. Now set $v_{2}(z)=v_{1}(c z)$, where $c$ is a positive constant. Then $B\left(r, v_{2}\right)=B\left(c r, v_{1}\right)$ so that

$$
\lim _{r \rightarrow \infty} \frac{B\left(r, v_{2}\right)-\sigma(\log r)^{2}}{\log r}=\alpha+2 \sigma \log c
$$

and we choose $c$ so that condition (i) obtains. Moreover

$$
A\left(r, v_{2}\right)=B\left(r, v_{2}\right)-\pi^{2} \sigma
$$

for all large $r$, say $r \geqslant R_{0}$, and (3.4) holds for $v_{2}$.

Since (3.4) holds we conclude that, given any positive number $M$, there is some number $r_{0}>R_{0}+2$ such that

$$
B\left(2 r_{0}, v_{2}\right)-B\left(r_{0}, v_{2}\right)>M \log r_{0} .
$$

For if there were no such $r_{0}$ we should have, for all large positive integers $n$,

$$
\begin{aligned}
B\left(2^{n}, v_{2}\right) & =B\left(2^{n}, v_{2}\right)-B\left(2^{n-1}, v_{2}\right)+B\left(2^{n-1}, v_{2}\right) \\
& \leqslant M(n-1) \log 2+B\left(2^{n-1}, v_{2}\right)-B\left(2^{n-2}, v_{2}\right)+B\left(2^{n-2}, v_{2}\right) \\
& \leqslant \ldots \leqslant \frac{1}{2} n^{2} M \log 2+O(1)=O\left(\log 2^{n}\right)^{2} .
\end{aligned}
$$

Thus $B\left(r, v_{2}\right)=O(\log r)^{2}$ and so $B(r, u)=O(\log r)^{2}$. This together with (2.6) contradicts (3.4).

Now $w(z)$ defined by

$$
\begin{aligned}
& w(z)=0, \quad|z| \leqslant 1, \\
& w(z)=\max \left\{\sigma(\log |z|)^{2}-\sigma(\operatorname{Arg} z)^{2}, 0\right\}, \quad|z|>1,
\end{aligned}
$$

is subharmonic in the plane (as was shown in the example following Theorem 1). We define a new function

$$
U(z)=\left\{\begin{array}{l}
w(z)+D, \quad|z| \leqslant r_{0} \\
h(z), \quad r_{0} \leqslant|z| \leqslant 2 r_{0} \\
v_{2}(z), \quad|z| \geqslant 2 r_{0}
\end{array}\right.
$$


where $D=B\left(r_{0}, v_{2}\right)-\sigma\left(\log r_{0}\right)^{2}+\pi^{2} \sigma$ and $h(z)$ is the harmonic function in $r_{0}<$ $|z|<2 r_{0}$ taking boundary values $w(z)+D$ on $|z|=r_{0}, v_{2}(z)$ on $|z|=2 r_{0}$. Clearly $U(z)$ satisfies conditions (i) and (ii) of Lemma 2. Moreover, once we have shown that $U(z)$ is subharmonic, it is evident that (3.7) holds.

To show that $U$ is subharmonic it is enough to show that $h$ dominates both $v_{2}$ and $w+D$ for $r_{0} \leqslant|z| \leqslant 2 r_{0}$. For $|z|=r_{0}$,

$$
h(z)=w(z)+D \geqslant \sigma\left(\log r_{0}\right)^{2}-\pi^{2} \sigma+D=B\left(r_{0}, v_{2}\right) \geqslant v_{2}(z),
$$

while for $|z|=2 r_{0}$,

$$
\begin{aligned}
h(z) & =v_{2}(z) \geqslant A\left(2 r_{0}, v_{2}\right)=B\left(2 r_{0}, v_{2}\right)-\pi^{2} \sigma>B\left(r_{0}, v_{2}\right)+M \log r_{0}-\pi^{2} \sigma \\
& =\sigma\left(\log r_{0}\right)^{2}+M \log r_{0}+D-2 \pi^{2} \sigma>\sigma\left(\log 2 r_{0}\right)^{2}+D \geqslant W(z)+D
\end{aligned}
$$

provided $M$ is large enough. This completes the proof.

4. Modification of $U$. Let $U$ be the function of Lemma 2. From (3.7) it follows that given any positive number $K$ there are arbitrarily large values of $r$ such that $B(r, U)>K(\log r)^{2}$. Given one such value, say $r_{1}$, take $2 R$ to be the smallest number greater than $r_{1}$ such that

$$
\frac{\sigma(\log 2 R)^{2}-B(2 R, U)}{\log 2 R}=A .
$$

From (3.5) such an $R$ exists; and clearly (if $K$ is large enough) $r_{1}<R$ so that

$$
\frac{\sigma(\log R)^{2}-B(R, U)}{\log R}<A \text {. }
$$

That is

$$
B(R, U)>\sigma(\log R)^{2}-A \log R .
$$

Also

$$
B(R, U)<B(2 R, U)=\sigma(\log 2 R)^{2}-A \log 2 R .
$$

We modify $U(z)$ in $|z|>R$ so as to obtain a subharmonic function which is not too large when $|z|$ is large. Let $h(z)$ be the harmonic function in $R<|z|<2 R$ which takes boundary values $U(z)$ on $|z|=R$ and $B(2 R, U)$ on $|z|=2 R$. This function clearly dominates $U$ on $R \leqslant|z| \leqslant 2 R$ so that

$$
U_{1}(z)= \begin{cases}U(z), & |z| \leqslant R, \\ h(z), & R \leqslant|z| \leqslant 2 R,\end{cases}
$$

is subharmonic in $|z|<2 R$. Further, $h(z) \geqslant h_{1}(z)$, where $h_{1}(z)$ is the harmonic function in $R<|z|<2 R$ taking boundary values $V_{1}=\sigma(\log R)^{2}-A \log R-$ $\pi^{2} \sigma$ on $|z|=R$ and $V_{2}=B(2 R, U)$ on $|z|=R$. (This follows from (3.6) and (4.2).) $h_{1}(z)$ may be written explicitly as

$$
h_{1}(z)=\frac{V_{2}-V_{1}}{\log 2} \log \frac{|z|}{R}+V_{1} \text {. }
$$

We define

$$
U_{2}(z)=2 \sigma(\log r)^{2}+B(2 R, U)-2 \sigma(\log 2 R)^{2}
$$


and show that, if $R$ is large, $U_{2}(z) \leqslant h(z)$ for $R<|z| \leqslant 2 R$ so that

$$
W_{R}(z)= \begin{cases}U_{1}(z), & |z| \leqslant 2 R, \\ U_{2}(z), & z \geqslant 2 R,\end{cases}
$$

is subharmonic. For $R<|z|<2 R$ we obtain, after some simplification,

$$
\begin{aligned}
h(z)-U_{2}(z) & \geqslant h_{1}(z)-U_{2}(z) \\
& =\left(\frac{B(2 R, U)+A \log R+\pi^{2} \sigma-\sigma(\log R)^{2}}{\log 2}-2 \sigma \log 2|z| R\right) \log \frac{|z|}{2 R} \\
& \geqslant\left(\frac{\sigma(\log 2 R)^{2}-A \log 2+\pi^{2} \sigma-\sigma(\log R)^{2}}{\log 2}-2 \sigma \log 2 R^{2}\right) \log \frac{|z|}{2 R} \\
& >0
\end{aligned}
$$

if $R$ is large enough.

We note that $W_{R}(z)=U_{1}(z)=U(z)$ for $|z| \leqslant R$ and that

$$
B\left(R^{2}, W_{R}\right)=W_{R}\left(R^{2}\right)<10 \sigma(\log R)^{2} .
$$

5. Behaviour of $\mu^{*}(r)$. Throughout this section the functions $A, B, \mu$ and $\mu^{*}$ will be understood to refer to the function $W_{R}$. Set $\rho=R^{3 / 2}$. Given $t \leqslant \frac{1}{2} R$ we have from (2.5)

$$
\begin{aligned}
& A(t)=\int_{|\zeta|<\rho} \log \left|1-\frac{z_{1}}{\zeta}\right| d \mu(\zeta)+O\left(\frac{t}{\rho} B(2 \rho)\right), \\
& B(t)=\int_{|\zeta|<\rho} \log \left|1-\frac{z_{2}}{\zeta}\right| d \mu(\zeta)+O\left(\frac{t}{\rho} B(2 \rho)\right),
\end{aligned}
$$

where $\left|z_{1}\right|=\left|z_{2}\right|=t$, and thus

$$
\begin{aligned}
A(t)-B(t)= & \int_{|\zeta|<R} \log \left|1-\frac{z_{1}}{\zeta}\right| d \mu(\zeta)-\int_{|\zeta|<R} \log \left|1-\frac{z_{2}}{\zeta}\right| d \mu(\zeta) \\
& +\int_{R<|\zeta|<\rho} \log \left|\frac{\zeta-z_{1}}{\zeta-z_{2}}\right| d \mu(\zeta)+O\left(\frac{t}{\rho} B(2 \rho)\right) \\
\geqslant & A_{1}(t, R)-B_{1}(t, R)-\int_{R}^{\rho} \log \left|\frac{s+t}{s-t}\right| d \mu^{*}(s)+O\left(\frac{t}{\rho} B(2 \rho)\right) \\
\geqslant & A_{2}(t, R)-B_{2}(t, R)-\mu^{*}(\rho) \log \left(\frac{R+t}{R-t}\right)+O\left(\frac{t}{\rho} B(2 \rho)\right) .
\end{aligned}
$$

Hence, for $1<R_{1}<R_{2}=\frac{1}{2} R$,

$$
\begin{aligned}
\Delta\left(R_{1}, R_{2}\right)= & \int_{R_{1}}^{R_{2}} \frac{d s}{s} \int_{0}^{s} \frac{A(t)-B(t)}{t} d t \\
\geqslant & \int_{R_{1}}^{R_{2}} \frac{d s}{s} \int_{0}^{s} \frac{A_{2}(t, R)-B_{2}(t, R)}{t} d t \\
& -\mu^{*}(\rho) \int_{R_{1}}^{R_{2}} \frac{d s}{s} \int_{0}^{s} \frac{1}{t} \log \left(\frac{R+t}{R-t}\right) d t+o\left(\frac{R_{2}}{\rho} B(2 \rho)\right) \\
= & T_{1}-T_{2}+o(1),
\end{aligned}
$$


from (4.6), and we estimate $T_{1}$ and $T_{2}$ in turn. From Lemma 1

$$
\begin{aligned}
T_{1}= & \mu^{*}(R) \int_{R_{1}}^{R_{2}} \frac{1}{s} d s \int_{0}^{\pi} \operatorname{Arg}\left(1-\frac{s}{R} e^{i \theta}\right) d \theta \\
& -\int_{0}^{R} \frac{\mu^{*}(t)}{t} d t \int_{R_{1}}^{R_{2}} \frac{1}{s} \log \left|\frac{t+s}{t-s}\right| d s .
\end{aligned}
$$

Also

$$
\operatorname{Arg}\left(1-\frac{S}{R} e^{i \theta}\right) \geqslant-\operatorname{Arcsin} \frac{S}{R} \geqslant-\frac{\pi S}{2 R}
$$

so

$$
T_{1} \geqslant-\frac{1}{4} \pi^{2} \mu^{*}(R)-I\left(R_{2}\right)+I\left(R_{1}\right)
$$

where

$$
I(r)=\int_{0}^{R} \frac{\mu^{*}(t)}{t} d t \int_{0}^{r} \frac{1}{s} \log \left|\frac{t+s}{t-s}\right| d s .
$$

We have

$$
\begin{aligned}
I\left(R_{2}\right) & \leqslant \int_{0}^{R} \frac{\mu^{*}(t)}{t} d t \int_{0}^{\infty} \frac{1}{s} \log \left|\frac{t+s}{t-s}\right| d s \\
& =\int_{0}^{R} \frac{\mu^{*}(t)}{t} d t \int_{0}^{\infty} \frac{1}{s} \log \left|\frac{s+1}{s-1}\right| d s \\
& =\frac{1}{2} \pi^{2} \int_{0}^{R} \frac{\mu^{*}(t)}{t} d t \leqslant \frac{1}{2} \pi^{2} B(R)+O(1),
\end{aligned}
$$

from (2.6), and combining this with (5.2) we obtain

$$
T_{1} \geqslant-\frac{1}{4} \pi^{2} \mu^{*}(R)-\frac{1}{2} \pi^{2} B(R)+I\left(R_{1}\right)+O(1) .
$$

A straightforward estimate yields

$$
T_{2}=\mu^{*}(\rho) \int_{R_{1} / R}^{R_{2} / R} \frac{d s}{s} \int_{0}^{s} \frac{1}{t} \log \left(\frac{1+t}{1-t}\right) d t \leqslant 2 \mu^{*}(\rho)
$$

and combining (5.1), (5.4) and (5.5) we obtain

$$
\begin{aligned}
\Delta\left(R_{1}, R_{2}\right) & \geqslant-\left(2+\frac{1}{4} \pi^{2}\right) \mu^{*}(\rho)-\frac{1}{2} \pi^{2} B(R)+I\left(R_{1}\right)+O(1) \\
& \geqslant-\frac{\left(4+\frac{1}{2} \pi^{2}\right)}{\log R} \int_{R^{3 / 2}}^{R^{2}} \frac{\mu^{*}(t)}{t} d t-\frac{1}{2} \pi^{2} B(R)+I\left(R_{1}\right)+O(1) \\
& \geqslant-\frac{10}{\log R} B\left(R^{2}\right)-\frac{1}{2} \pi^{2} B(R)+I\left(R_{1}\right)+O(1) \\
& \geqslant-110 \sigma \log R-\frac{1}{2} \pi^{2} B(R)+I\left(R_{1}\right),
\end{aligned}
$$

from (4.6). On the other hand $W(z)=U(z)$ for $|z| \leqslant R$ and thus from (3.6)

$$
\begin{aligned}
\Delta\left(R_{1}, R_{2}\right) & \leqslant \int_{R_{1}}^{R_{2}}\left(\int_{e^{\pi}}^{s} \frac{-\pi^{2} \sigma}{t} d t+\int_{r_{0}}^{2 r_{0}} \frac{\pi^{2} \sigma}{t} d t\right) \frac{d s}{s} \\
& \leqslant-\frac{1}{2} \pi^{2} \sigma\left(\log R_{2}\right)^{2}+\frac{1}{2} \pi^{2} \sigma\left(\log R_{1}\right)^{2}+2 \pi^{3} \sigma \log R_{2} .
\end{aligned}
$$


Since $2 \pi^{3}<70,(5.6)$ and (5.7) together yield

$$
\frac{1}{2} \pi^{2}\left(B(R)-\sigma\left(\log R_{2}\right)^{2}\right)+180 \sigma \log R \geqslant I\left(R_{1}\right)-\frac{1}{2} \pi^{2} \sigma\left(\log R_{1}\right)^{2} .
$$

Since $R_{2}=\frac{1}{2} R$ the left-hand side of (5.8) is, from (4.3), no larger than

$$
\begin{aligned}
& \frac{1}{2} \pi^{2}\left(\sigma(\log 2 R)^{2}-A \log 2 R-\sigma\left(\log \frac{1}{2} R\right)^{2}\right)+180 \sigma \log R \\
& =\log R\left(2 \pi^{2} \sigma \log 2-\frac{1}{2} A \pi^{2}+180 \sigma\right)-\frac{1}{2} \pi^{2} A \log 2<0
\end{aligned}
$$

when $R$ is large, from (3.5). Thus (5.8) reduces to

$$
\frac{1}{2} \pi^{2} \sigma\left(\log R_{1}\right)^{2}>I\left(R_{1}\right) \text {. }
$$

Now $W=U_{1}=U$ for $|z| \leqslant R$ and $U$ satisfies (3.7). Thus, by taking $R$ sufficiently large, we can find $R_{1}$ such that $1<R_{1}<R_{1}^{3 / 2}<\frac{1}{2} R$ and $\mu^{*}\left(R_{1}^{1 / 2}\right)>5 \sigma \log R_{1}$. For such an $R_{1}$

$$
\begin{aligned}
I\left(R_{1}\right) & \geqslant \int_{R_{1}^{1 / 2}}^{R_{1}^{3 / 2}} \frac{\mu^{*}(t)}{t} d t \int_{0}^{R_{1}} \frac{1}{s} \log \left|\frac{t+s}{t-s}\right| d s \\
& \geqslant \mu^{*}\left(R_{1}^{1 / 2}\right) \int_{R_{1}^{1 / 2}}^{R_{1}^{3 / 2}} \frac{1}{t} d t \int_{0}^{R_{1}} \frac{1}{s} \log \left|\frac{t+s}{t-s}\right| d s \\
& >5 \sigma \log R_{1} \int_{R_{1}^{-1 / 2}}^{R_{1}^{1 / 2}} \frac{1}{t} d t \int_{0}^{1 / t} \frac{1}{s} \log \left|\frac{s+1}{s-1}\right| d s \\
& >5 \sigma \log R_{1} \int_{R_{1}^{-1 / 2}}^{1} \frac{1}{t} d t \int_{0}^{1} \frac{1}{s} \log \left|\frac{s+1}{s-1}\right| d s \\
& =\frac{5 \sigma}{8} \pi^{2}\left(\log R_{1}\right)^{2},
\end{aligned}
$$

which contradicts (5.9). The assumption which leads to this contradiction, namely (3.4), is thus mistaken and we deduce (3.3).

6. Bounds on the growth of $B(r)$. The remainder of the paper is concerned only with the functions $u$ and $u_{1}$ occurring in the statement of the theorems. In this section we aim to show that (3.1) and (3.2) together imply

$$
-\infty<\alpha=\lim _{r \rightarrow \infty} \frac{B(r)-\sigma(\log r)^{2}}{\log r}<\beta=\varlimsup_{r \rightarrow \infty} \frac{B(r)-\sigma(\log r)^{2}}{\log r}<+\infty .
$$

As we have shown, (3.3) holds and we may thus introduce

$$
u_{1}(z)=\lim _{R \rightarrow \infty} u_{2}(z, R)=\operatorname{Re}\left(z \int_{0}^{\infty} \frac{\mu^{*}(t)}{t(t+z)} d t\right) .
$$

We write $B_{1}(r)=\max _{|z|=r} u_{1}(z), A_{1}(r)=\inf _{|z|=r} u_{1}(z)$ and take the limit as $R \rightarrow \infty$ in the first equation of Lemma 1 to obtain for all large $R_{1}<R_{2}$

$$
0 \geqslant \int_{R_{1}}^{R_{2}} \frac{A_{1}(t)-B_{1}(t)+\pi^{2} \sigma}{t} d t=\nu\left(R_{1}\right)-\nu\left(R_{2}\right),
$$

where

$$
\nu(r)=\int_{0}^{\infty} \frac{\mu^{*}(t)}{t} \log \left|\frac{t+r}{t-r}\right| d t-\pi^{2} \sigma \log r
$$


We conclude that $\nu(r)$ is nondecreasing for all large $r$. Integrating $(6.3)$ over $(1, r)$ we obtain

so that

$$
\int_{0}^{\infty} \frac{\mu^{*}(t)}{t} d t \int_{1}^{r} \frac{1}{s} \log \left|\frac{t+s}{t-s}\right| d s-\frac{1}{2} \pi^{2} \sigma(\log r)^{2}=\int_{1}^{r} \frac{\nu(s)}{s} d s
$$

$$
\int_{0}^{\infty} \frac{\mu^{*}(t)}{t} d t \int_{0}^{r} \frac{1}{s} \log \left|\frac{t+s}{t-s}\right| d s-\frac{1}{2} \pi^{2} \sigma(\log r)^{2}=\int_{1}^{r} \frac{\nu(s)}{s}+O(1)
$$

Thus

where

$$
\begin{aligned}
B_{1}(r) & =r \int_{0}^{\infty} \frac{\mu^{*}(t)}{t(t+r)} d t \\
& =\sigma(\log r)^{2}+\frac{2}{\pi^{2}} \int_{1}^{r} \frac{\nu(t)}{t} d t+J(r)+O(1)
\end{aligned}
$$

$$
J(r)=\int_{0}^{\infty} \frac{\mu^{*}(t)}{t}\left(\frac{r}{r+t}-\frac{2}{\pi^{2}} \int_{0}^{r} \frac{1}{s} \log \left|\frac{s+t}{s-t}\right| d s\right) d t .
$$

Two changes of variable in the integrals of (6.6) yield

where

$$
J(r)=\int_{0}^{\infty} \frac{\mu^{*}(r t)}{t} h(t) d t
$$

$$
h(t)=\frac{1}{1+t}-\frac{2}{\pi^{2}} \int_{0}^{1 / t} \frac{1}{s} \log \left|\frac{1+s}{1-s}\right| d s .
$$

We have

LEMMA 3. $h(t) \geqslant 0$ for $t \geqslant 1$ and $h(1 / t)=-h(t)$ for $t \geqslant 1$.

For $t>1$

$$
\begin{aligned}
(t+1) \frac{2}{\pi^{2}} \int_{0}^{1 / t} \frac{1}{s} \log \left|\frac{1+s}{1-s}\right| d s & =(t+1) \frac{2}{\pi^{2}} \int_{0}^{1 / t}\left(2+\frac{2 s^{2}}{3}+\frac{2 s^{4}}{5}+\ldots\right) d s \\
& =(t+1) \frac{4}{\pi^{2}}\left(\frac{1}{t}+\frac{1}{3^{2} t^{3}}+\frac{1}{5^{2} t^{5}}+\ldots\right) \\
& =\frac{4}{\pi^{2}}\left(1+\frac{1}{t}+\frac{1}{3^{2} t^{2}}+\frac{1}{3^{2} t^{3}}+\ldots\right) \\
& <\frac{8}{\pi^{2}}\left(1+\frac{1}{3^{2}}+\frac{1}{5^{2}}+\ldots\right)=1 .
\end{aligned}
$$

Thus $h(t)>0$ for $t>1$ and certainly $h(1)=0$. Further, for $t>1$,

$$
\begin{aligned}
h\left(\frac{1}{t}\right) & =\frac{t}{1+t}-\frac{2}{\pi^{2}} \int_{0}^{t} \frac{1}{s} \log \left|\frac{s+1}{s-1}\right| d s \\
& =\frac{t}{1+t}-\frac{2}{\pi^{2}} \int_{1 / t}^{\infty} \frac{1}{s} \log \left|\frac{s+1}{s-1}\right| d s \\
& =\frac{t}{1+t}-\frac{2}{\pi^{2}}\left(\frac{\pi^{2}}{2}-\int_{0}^{1 / t} \frac{1}{s} \log \left|\frac{s+1}{s-1}\right| d s\right)=-h(t)
\end{aligned}
$$

and this proves Lemma 3. 
Now, from (6.7) and Lemma 3,

$$
\begin{aligned}
J(r) & =\int_{0}^{1} \frac{\mu^{*}(r t)}{t} h(t) d t+\int_{1}^{\infty} \frac{\mu^{*}(r t)}{t} h(t) d t \\
& =\int_{1}^{\infty} \frac{\mu^{*}(r / t)}{t} h(1 / t) d t+\int_{1}^{\infty} \frac{\mu^{*}(r t)}{t} h(t) d t \\
& =\int_{1}^{\infty} \frac{\mu^{*}(r t)-\mu^{*}(r / t)}{t} h(t) d t \geqslant 0 .
\end{aligned}
$$

Combining this with (6.5) we obtain

$$
B_{1}(r) \geqslant \sigma(\log r)^{2}+\frac{2}{\pi^{2}} \int_{1}^{r} \frac{\nu(t)}{t} d t+O(1)
$$

Hence

$$
\alpha_{1}=\lim _{r \rightarrow \infty} \frac{B_{1}(r)-\sigma(\log r)^{2}}{\log r} \geqslant \frac{\lim }{r \rightarrow \infty}\left(\frac{2}{\pi^{2} \log r} \int_{1}^{r} \frac{\nu(t)}{t} d t\right)>-\infty,
$$

since $\nu(t)$ is nondecreasing. Moreover, since for any $k>1$

$$
\begin{aligned}
B_{1}(r) & =r \int_{0}^{\infty} \frac{\mu^{*}(t)}{t(t+r)} d t \\
& \leqslant \int_{0}^{k r} \frac{\mu^{*}(t)}{t} d t+O\left(r \int_{k r}^{\infty} \frac{\log t}{t(t+r)} d t\right) \\
& \leqslant B(k r)+O\left(\int_{k}^{\infty} \frac{\log r+\log t}{t(t+1)} d t\right)=B(k r)+O(\log r)
\end{aligned}
$$

we deduce at once from (6.9) that

$$
\alpha=\lim _{r \rightarrow \infty} \frac{B(r)-\sigma(\log r)^{2}}{\log r}>-\infty .
$$

But $\alpha<\infty$ by hypothesis and from this together with (6.10) we deduce that $\alpha_{1}<\infty$. Thus, from (6.9),

$$
\lim _{r \rightarrow \infty} \nu(t)=A_{0}
$$

exists and is finite. From (6.5), then,

$$
\beta_{1}=\varlimsup_{r \rightarrow \infty} \frac{B_{1}(r)-\sigma(\log r)^{2}}{\log r}=\frac{2}{\pi^{2}} A_{0}+\varlimsup_{r \rightarrow \infty} \frac{J(r)}{\log r} .
$$

But

$$
\begin{aligned}
J(r) & =\int_{1}^{\infty} \frac{1}{t}\left(\mu^{*}(r t)-\mu^{*}(r / t)\right) h(t) d t \leqslant \int_{1}^{\infty} \frac{\mu^{*}(r t)}{t(1+t)} d t \\
& =O\left(\int_{1}^{\infty} \frac{\log t+\log r}{t(1+t)} d t\right)=O(\log r)
\end{aligned}
$$

so, from (6.12), $\beta_{1}<\infty$. Since $\beta \leqslant \beta_{1}$ we deduce finally (6.1). 


\section{Conclusion of the proofs of Theorems 1 and 2. We prove}

LEMMA 4. $\mu^{*}(r)=2 \sigma \log r+O(1)$.

Since $\lim _{r \rightarrow \infty} \nu(r)$ exists and is finite we deduce from (6.2) that

Hence

$$
\int_{1}^{\infty} \frac{A_{1}(t)-B_{1}(t)+\pi^{2} \sigma}{t} d t>-\infty
$$

$$
A_{1}(r)>B_{1}(r)-2 \pi^{2} \sigma
$$

outside a set $E$ of finite logarithmic measure, and thus

$$
\begin{aligned}
\int_{0}^{r} \frac{\mu^{*}(t)}{t} d t & =\frac{1}{2 \pi} \int_{0}^{2 \pi} u_{1}\left(r e^{i \theta}\right) d \theta=B_{1}(r)+O(1) \\
& =\int_{0}^{\infty} \frac{r \mu^{*}(t)}{t(t+r)} d t+O(1)
\end{aligned}
$$

as $r \rightarrow \infty$ outside $E$. Rearranging (7.2) yields

$$
\int_{1}^{\infty} \frac{\mu^{*}(r t)-\mu^{*}(r / t)}{t(t+1)} d t=O(1)
$$

Using (6.8) and the fact that $h(t) \leqslant 1 /(1+t)$, we deduce that $J(r)=O(1)$, and so (6.5) yields

$$
B_{1}(r)=\sigma(\log r)^{2}+\frac{2}{\pi^{2}} \int_{1}^{r} \frac{\nu(t)}{t} d t+O(1)
$$

as $r \rightarrow \infty$ outside $E$. Combining (7.2) and (7.3) we obtain

$$
\int_{0}^{r} \frac{\mu^{*}(t)}{t} d t=\sigma(\log r)^{2}+\frac{2}{\pi^{2}} \int_{1}^{r} \frac{\nu(t)}{t} d t+O(1)
$$

as $r \rightarrow \infty$ outside $E$. Now, given $r$ outside $E$, we may choose $k$ satisfying $3>k>2$ such that $k r$ is also outside $E$ (this follows since $E$ has finite logarithmic measure). Thus, for $r$ outside $E$,

$$
\begin{aligned}
\mu^{*}(r) \log k & \leqslant \int_{r}^{k r} \frac{\mu^{*}(t)}{t} d t \\
& =2 \sigma \log k \log r+\sigma(\log k)^{2}+\frac{2}{\pi^{2}} \int_{r}^{k r} \frac{\nu(t)}{t} d t+O(1) \\
& \leqslant 2 \sigma \log k \log r+\sigma(\log k)^{2}+\frac{2}{\pi^{2}} \nu(k r) \log k+O(1)
\end{aligned}
$$

and so

$$
\mu^{*}(r) \leqslant 2 \sigma \log r+O(1)
$$

for $r$ outside $E$. Since $E$ has finite logarithmic measure and $\mu^{*}(r)$ increases with $r$ we easily deduce that (7.4) holds for all large $r$. Quite similarly we obtain $\mu^{*}(r) \geqslant 2 \sigma \log r+O(1)$ and this proves the lemma.

From Lemma 4 and (6.8) it follows that $J(r)=O(1)$ as $r \rightarrow \infty$ and hence, from (6.5), $\alpha_{1}=\beta_{1}$. From (7.1)

$$
B(r)>A(r)>A_{1}(r)>B_{1}(r)-2 \pi^{2} \sigma
$$


for $r$ outside $E$. But $E$ has finite logarithmic measure and so, given any $r$, we may choose $k=k(r)>1$ such that $r / k$ is outside $E$ and $k(r) \rightarrow 1$ as $r \rightarrow \infty$. Hence $B(r)>B(r / k)>B_{1}(r / k)+O(1)$ and finally

$$
\begin{aligned}
\alpha & =\frac{\lim _{r \rightarrow \infty}}{B(r)-\sigma(\log r)^{2}} \\
& =\alpha_{1}-\lim _{r \rightarrow \infty} 2 \sigma \log k=\alpha_{1 \rightarrow \infty} .
\end{aligned}
$$

But $\alpha \leqslant \beta \leqslant \beta_{1}=\alpha_{1}$ so $\alpha=\beta=\alpha_{1}=\beta_{1}$ and this completes the proof of Theorems 1 and 2. Let us note that, from (7.3), $\alpha=\left(2 / \pi^{2}\right) A_{0}$.

8. Proof of Theorem 3. We write $\mu^{*}(r)=2 \sigma \log r+\varepsilon(r)$, where $\varepsilon(r)$ is bounded for $r \geqslant 1$. From (6.3) we obtain

$$
\int_{0}^{\infty} \frac{\mu^{*}(t)-2 \sigma \log t}{t} \log \left|\frac{t+r}{t-r}\right| d t=\nu(r) .
$$

We introduce $\varepsilon_{1}(t)=\varepsilon(t)$ for $t \geqslant 1, \varepsilon_{1}(t)=\varepsilon(1)$ for $0 \leqslant t \leqslant 1$, and write

$$
\nu_{1}(r)=\nu(r)+\int_{0}^{1}(\varepsilon(1)-\varepsilon(t)) \log \left|\frac{t+r}{t-r}\right| d t / t .
$$

Then $\nu_{1}(r) \rightarrow A_{0}$ as $r \rightarrow \infty$ and (8.1) may be rewritten as

$$
\int_{0}^{\infty} \frac{\varepsilon_{1}(t)}{t} \log \left|\frac{t+r}{t-r}\right| d t=\nu_{1}(r)
$$

With a change of variable we obtain

$$
\int_{-\infty}^{\infty} \varepsilon_{1}\left(e^{t}\right) \log \left|\frac{e^{r-t}+1}{e^{r-t}-1}\right| d t=\nu_{1}\left(e^{r}\right) .
$$

Now $\varepsilon_{1}\left(e^{t}\right)=\mu^{*}\left(e^{t}\right)-2 \sigma t$ so

$$
\lim _{\substack{x \rightarrow \infty \\ y>x \\(y-x) \rightarrow 0}}\left(\varepsilon_{1}\left(e^{y}\right)-\varepsilon_{1}\left(e^{x}\right)\right) \geqslant 0
$$

that is, $\varepsilon_{1}\left(e^{t}\right)$ is slowly decreasing in the sense of $[8$, p. 209]. Moreover an application of contour integration yields

$$
\int_{-\infty}^{\infty} e^{i x t} \log \left|\frac{e^{t}+1}{e^{t}-1}\right| d t=\frac{\pi}{|x|} \frac{1-e^{-\pi|x|}}{1+e^{-\pi|x|}}
$$

for $x \neq 0$. We are thus able to apply Theorem $10 \mathrm{a}$ of $[8, \mathrm{p} .211]$, to deduce that

$$
\varepsilon_{1}\left(e^{t}\right) \rightarrow \frac{2 A_{0}}{\pi^{2}}=\alpha
$$

as $t \rightarrow \infty$ and this completes the proof of Theorem 3 .

NoTE ADDED IN PROOF. The conjecture that follows the statement of Theorem 1 is false. Details of a correct theorem for $p \neq 2$ should appear in due course. 


\section{REFERENCES}

1. P. D. Barry, The minimum modulus of small integral and subharmonic functions, Proc. London Math. Soc. 12 (1962), 445-495.

2. __ On a theorem of Kjellberg, Quart. J. Math. Oxford Ser. 15 (1964), 179-191.

3. P. C. Fenton, The infimum of small subharmonic functions, Proc. Amer. Math. Soc. 78 (1980), 43-47.

4. W. K. Hayman, The minimum modulus of large integral functions, Proc. London Math. Soc. 2 (1952), 469-512.

5. Bo Kjellberg, On the minimum modulus of entire functions of lower order less than one, Math. Scand. 8 (1960), 189-197.

6. $\ldots$ A theorem on the minimum modulus of entire functions, Math. Scand. 12 (1963), 5-11.

7. T. Rado, Subharmonic functions, Springer-Verlag, Berlin and New York, 1937.

8. D. V. Widder, The Laplace transform, Princeton Math. Ser., vol. 6, Princeton Univ. Press, Princeton, N. J., 1941.

Department of Mathematics, University of Otago, Dunedn, New Zealand 